\title{
The Usefulness of a 3D Whole-Body Scanner for Uncommon Cosmetic Surgery Procedures
}

\author{
David B. STEFAN ${ }^{1}$, David A. GILBERT ${ }^{2}$ \\ ${ }^{1}$ Novaptus Systems Inc., Chesapeake VA, USA; \\ ${ }^{2}$ The Hague Plastic and Cosmetic Surgery Center, Norfolk VA, USA \\ DOI: $10.15221 / 17.009$ http://dx.doi.org/10.15221/17.009
}

\section{Abstract \\ Background}

A 3D whole-body scanner appears to be best suited for measuring and documenting cosmetic surgical procedures that result in significant changes to the body. These procedures include breast augmentation, breast reduction and abdominoplasty. It is less useful for measuring changes resulting from procedures such as lipoplasty, which do not immediately result in pronounced changes of body contours. This paper presents examples of 3D whole-body scanning for less common cosmetic surgery procedures, and explores the usefulness of the 3D scanner as an aid for evaluating the pre-operative condition and as a tool for measuring and documenting post-operative changes of these surgeries.

\section{Methods}

A 3D whole-body scanner has been deployed for use in a cosmetic surgery practice in Norfolk, Virginia since 2002. Over the course of time, pre-operative and post-operative body scans from a wide range of cosmetic procedures have been collected. The 3D body scanner has shown its utility in capturing accurate whole-body models of subjects that underwent various surgical procedures. The scanner's measurement capabilities appear to be more effective for certain procedures and less useful for others. Case 1 examines circumferential pannulectomy. Case 2 examines a gluteal implant procedure. Case 3 examines a brachioplasty procedure. Case 4 examines chest contouring procedures.

\section{Discussion}

A general purpose, 3D whole-body scanner has certain limitations. It lacks definition for some regions of the body. This type of scanner is of little use for facial, hand or foot procedures, as these are outside the scanner's coverage area. In the case of a pannulectomy or gluteal implants, changes to the body are significant and the measurements are meaningful. The scanner provides less utility for procedures involving release of recessed body parts or removal of small growths. In these cases, pre-operative and post-operative measurements show little difference. The fixed position of the subject in the scan chamber limits the coverage for procedures related to the arms and neck. The 3D body scanner excels in coverage of the male chest region and is useful in chest contouring and gynecomastia procedures. The body scanner also has excellent coverage of the back side of the patient, including the buttocks region.

\section{Summary}

A 3D whole-body scanner has its place as a tool to document the pre-operative subject's body and measure overall dimensions. This has its own benefit as part of a 3D digital archive. In certain procedures, these body models and measurements can assist the surgeon in evaluating the subject's pre-operative body contours and act as an aid for planning the pending surgery. Post-operative measurements faithfully document the results of these surgeries. The 3D whole-body scanner is not as effective in measuring procedures that result in less pronounced changes to the body, such as lipoplasty, removal of small growths, or procedures that are beyond the coverage area of the scanner. A table at the end of this paper enumerates the type of cosmetic surgeries performed, and the surgeon's opinion as to the usefulness of the 3D scanner as an aid for pre-operative body contour evaluation or as a tool to measure and document post-operative surgical results. 


\section{Introduction}

IRB NUMBER 05-09-FB-0235 Eastern Virginia Medical School, Norfolk, Virginia, USA provides the basis for this research and presentation, to which the subjects have signed the appropriate consent forms.

For the background and history of the placement of the original 3D body scanner and subsequent model upgrades the reader is referred to papers found in the reference section. A summary follows.

A 3D whole-body booth-type scanner, in its original mechanical sensor head configuration and its solidstate sensor head successors, has been employed at The Hague Center for Cosmetic Surgery in Norfolk, Virginia since November of 2002.

During the span of these years, many patients have been scanned pre-operatively and post-operatively, often on a periodic basis as part of routine follow-up visits. The series of pre-operative and postoperative scans form the patient's 3D digital archive, of which a separate paper has been dedicated to discussing such an archive's importance (PAPER ID \#50, $8^{\text {th }}$ 3DBODY.TECH Conference).

The 3D scanner has a useful coverage area that focuses on the trunk, legs and arms of the individual within the scan chamber. Hands, feet and head imaging is intentionally degraded. As such, the 3D scanner has not been utilized for patients who underwent surgical procedures in these areas.

The practice encounters patients that are seeking aesthetic improvements through surgical procedures. Well-known procedures include breast augmentation, breast reduction, breast lift, abdominoplasty ("tummy-tuck") and liposuction. Liposuction is often an adjunct procedure performed in conjunction with another surgery. The reader is encouraged to review papers found in the reference section that specifically address the use of a 3D body scanner for measuring, evaluating and documenting these case examples.

Less common aesthetic surgical procedures include lower-body lifts, gluteal implants, chest contouring and brachioplasty (reduction and removal of pendulous tissue from the lower part of the arms.) These procedures are within the coverage area of the 3D scanner, and could presumably be measured preoperatively as an aid to body contour evaluation, and post-operatively to document the physical changes of the surgical outcome. Examples of these procedures, along with corresponding 3D body models and measurements are presented and discussed.

\section{Case 1. Circumferential Pannulectomy.}

A "lower-body lift" procedure is known as a "circumferential pannulectomy."

Figure 1 is an artist's illustration of the pre-operative markings a surgeon would make prior to the lowerbody lift procedure.
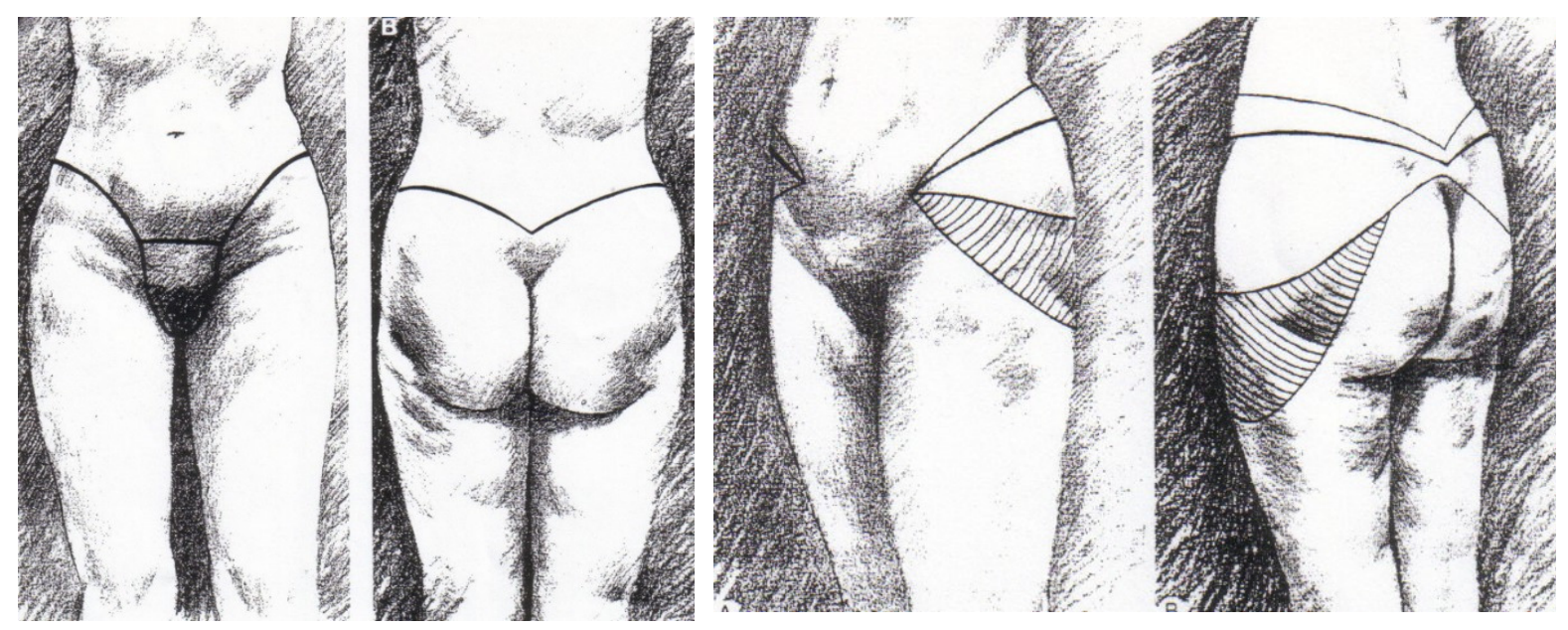

Figure 1. Artist's Rendering of Body Markings for a Lower-Body Lift 
The illustration on the left depicts the circumferential nature of the procedure. The illustration on the right indicates that the front of thigh and lower back act as fixation points. The result of this surgery is the appearance of a lift of the lower body.

Figure 2 displays pre- and post-operative posterior photographs of a patient that underwent a circumferential pannulectomy. One can observe the extent of the incisions made circumferentially to achieve the desired "lift" provided by this surgical procedure.
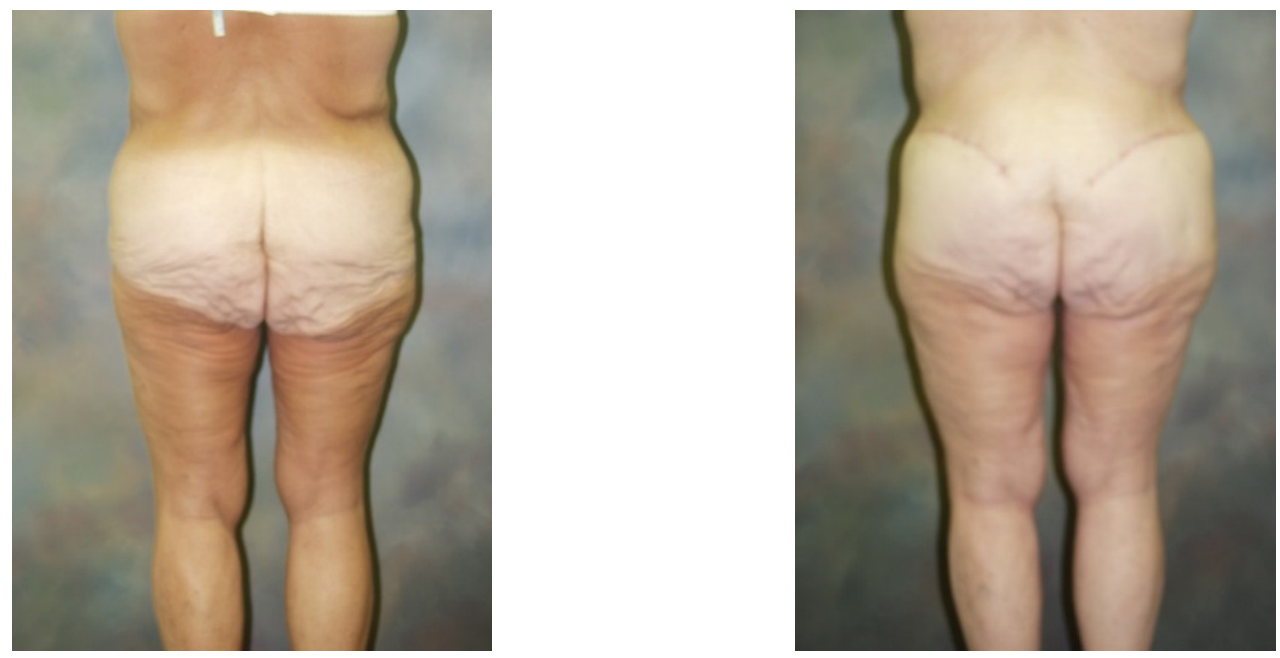

Figure 2. Pre- and Post-Operative Photos of the a Lower-Body Lift Procedure

Figure 3 depicts a more complex form of a circumferential pannulectomy is known as the "Fleur-di-lis" pannulectomy, so called for its pre-operative markings, which resemble that symbol.
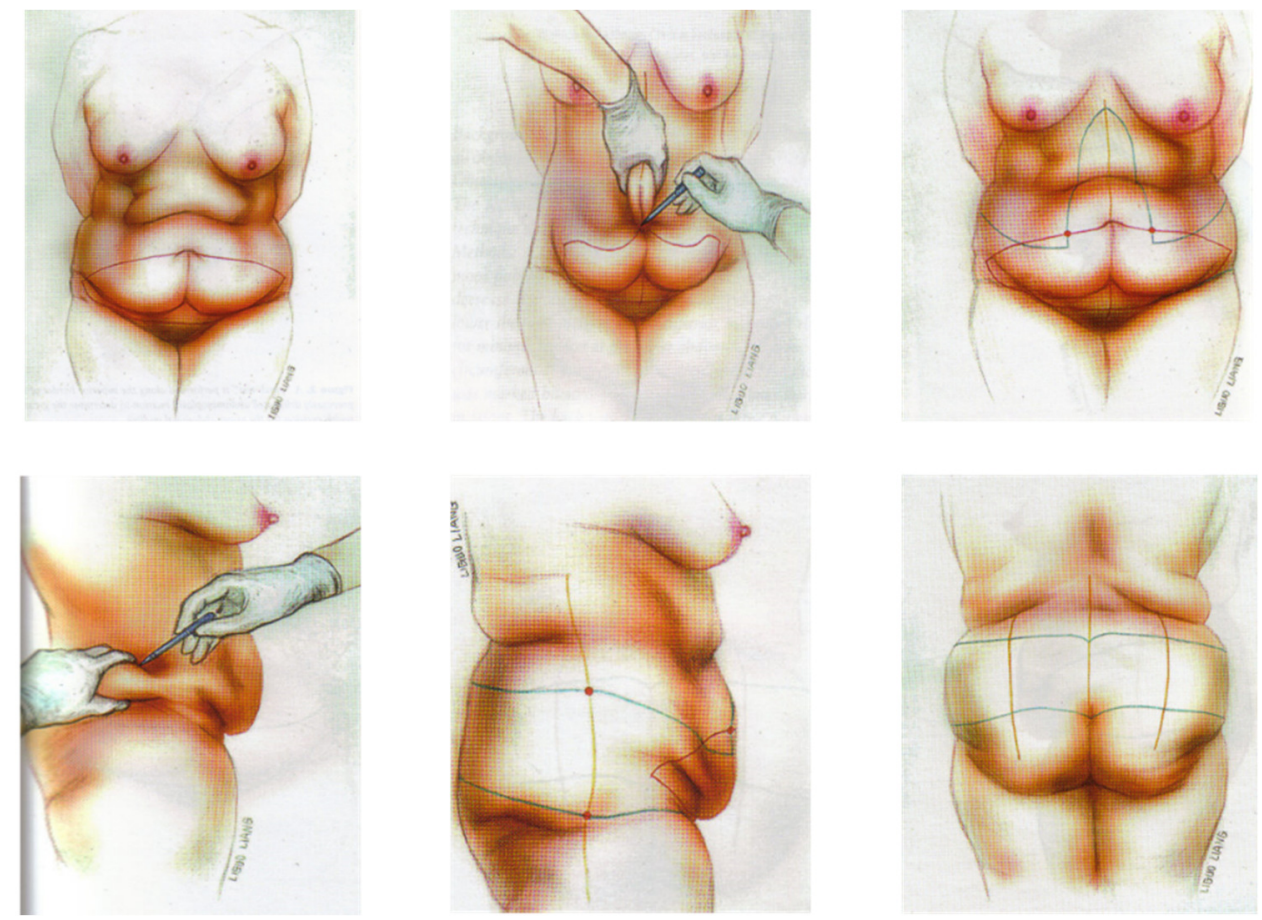

Figure 3. Body Markings for A "Fleur-di-lis" Circumferential Pannulectomy

Figure 4 presents images of a 3D body model of a pre-operative patient that has been marked according to this Fleur-di-lis pattern. 

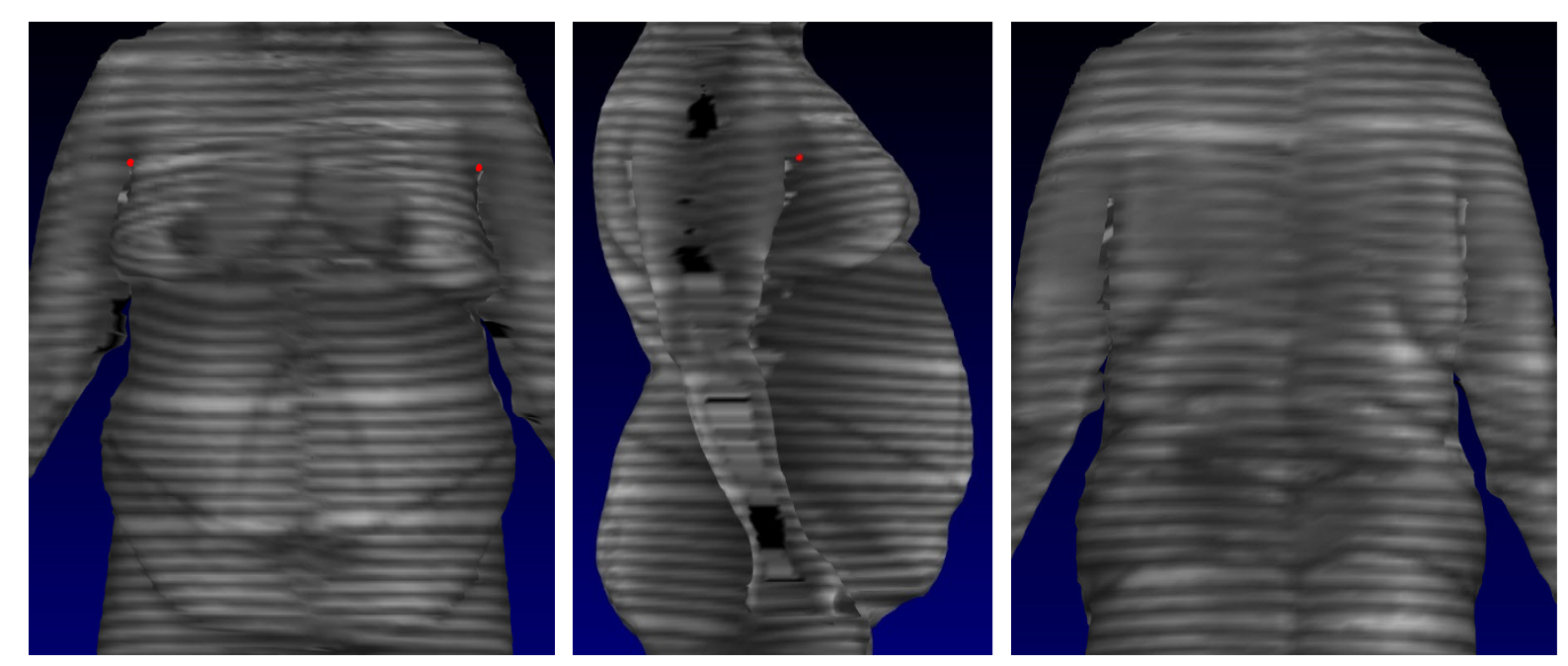

Figure 4. Image from a 3D Body Model of a Pre-Operative Patient with "Fleur-di-lis" Body Markings

The horizontal lines on these images represent the structured light pattern projected and captured by the sensors within the chamber of the 3D booth-type scanner, and are evident when the "color" display mode is selected (versions fielded between 2005 and 2012).

Figure 5 displays similar images from the 3D body model of the patient scanned post-operatively at 8 months.
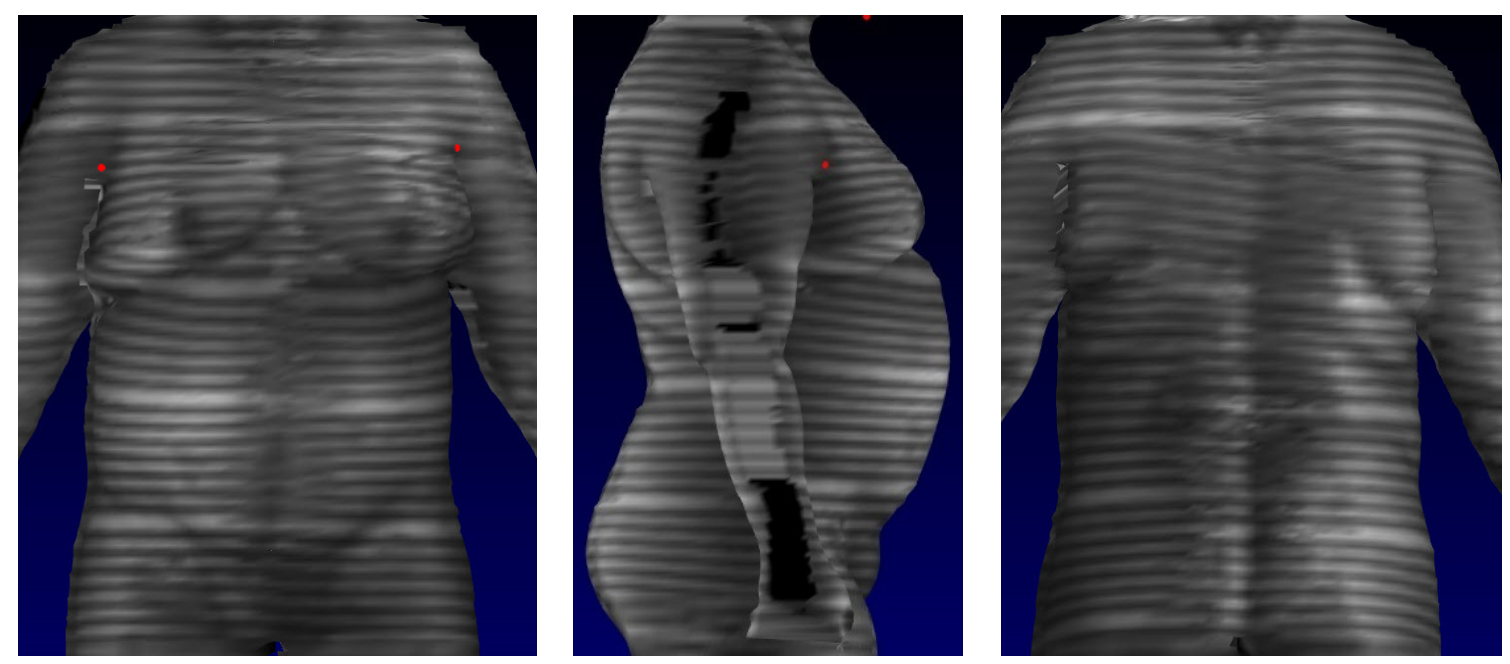

Figure 5. Post-Operative 3D Body Model Images

The 3D scanner and associated measurement software can be used to document the pre-operative dimensions of the patient. Figure 6 depicts a measurement template designed to document the lowerhalf of the body. The yellow lines indicate the locations of the measurement extractions. 

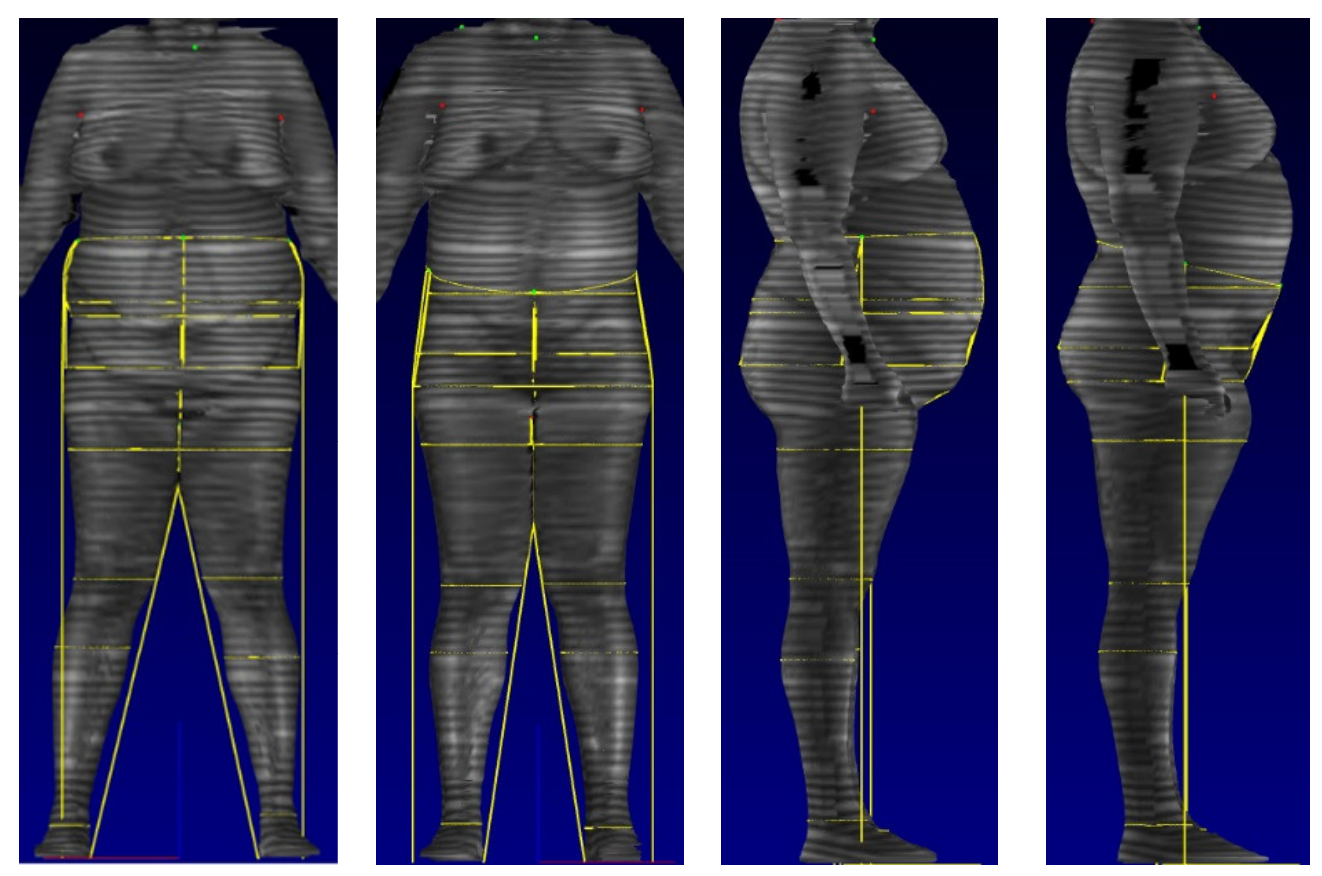

Figure 6. Pre- and Post-Operative 3D Body Model Images with Measurement Extraction Lines

Figure 7 presents the measurements produced by the programmable measurement extraction profile for the pre-operative and 8-month post-operative 3D body models.

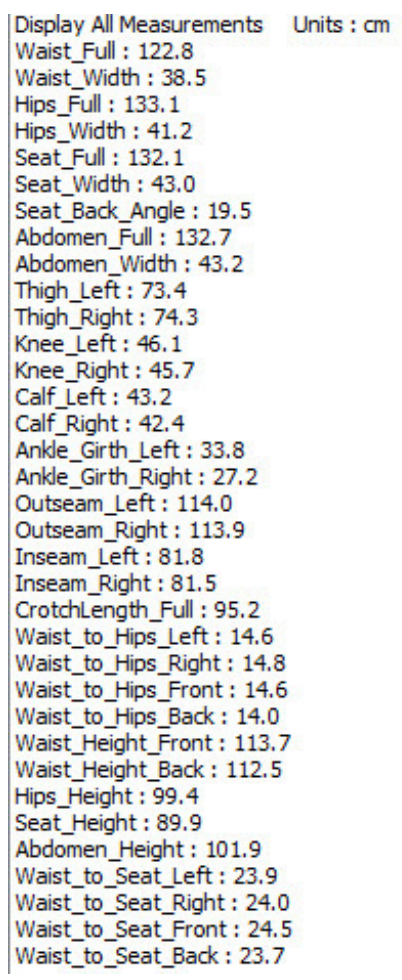

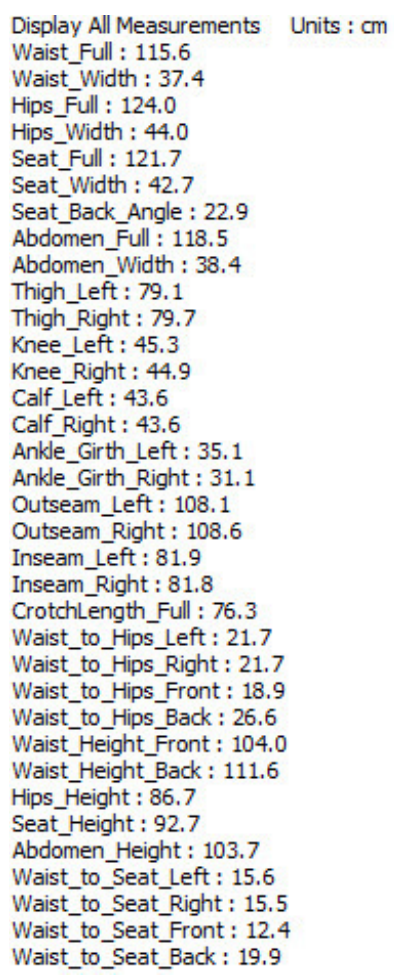

Figure 7. Pre- and Post-Operative Lower-Body Measurement Extractions

The pre- and post-operative measurement comparisons provide for an interesting discussion. The key landmark that the measurement software searches for on a 3D body model to extract lower body measurements is the "small of the back." The landmark appears consistent on each 3D body model displayed, as indicated by the similar heights of the yellow measurement line near the small of the back. Circumferential measurements such as waist, hips, seat and abdomen have been reduced. However, 
though the "small of the back" landmark's height appears consistent, the new body contours of the postoperative patient caused the measurement software to locate the hips height nearly $13 \mathrm{~cm}$ lower on the post-operative 3D body model, reflecting the "lift" of the body resulting from the surgery.

Measurements for custom garments, for which the scanner software was developed, is evident here. If one were to construct a garment for the pre-operative patient, the clothier would use the pre-operative measurements, including the location of the hips and its associated circumference. The garment, such as a pair of pants or a skirt, would fit this pre-operative body well. Conversely, contour changes resulting from the surgery affect the not only the apparel size, but the location of common measurements, such as the hips height and hips circumference required to construct a well-fitting custom garment.

The effect of this pannulectomy was to reduce the abdominal area and lift the lower body, particularly the buttocks. To the measurement software, this is the same as increasing the distance between the waist and hips while reducing both the waist and the hips circumference (resulting in a slimmer appearance).

Another interesting measurement is the "CrotchLength_Full" distance. This measurement line is constructed from the small of the back where the waist measurement begins, traveling under the "crotch" of the individual, terminating at the front medial point of the circumferential waist measurement. Presumably, this individual could now wear pants that had the characteristics of this line, with the front of the waistline lower in height than the back of the waistline.

The circumferential pannulectomy examples discussed so far are truly cosmetic in nature. The 3D body scanner displays adequate coverage, and the 3D body models acquired from scanning the patient can be measured with fidelity. These measurements can be recorded, retrieved and compared.

There are also many cases that aim to provide surgical relief to patients with conditions that are either painful, or cause a material inconvenience to the enjoyment of daily living. Such situations can concern hygiene, continual rashes or abrasion caused by overlapping skin folds, or a large pannus overhang that interferes with the function of voiding.

A pannus, which can appear like an apron that extends below the abdominal area, is often encountered on subjects that have undergone a surgical weight loss procedure. This type of surgery can result in massive weight reduction over a relatively short time duration. Depending on the physical condition of the individual, such massive weight loss can result in skin folds. The massive weight loss can also result in a distended pannus. Large skin folds as well as a distended pannus can be surgically removed.

Figure 8 presents photographs of an unusual circumferential pannulectomy case.

Here is a case where the individual's pannus overlaps the abdomen and extends below the thighs.

The 3D body scanner produces a 3D body model of the surface of the individual being scanned. It is incapable of penetrating the surface. The scanner measurement software creates a 3D body model from the images that are acquired from the cameras that are in the sensors. These sensors are located at various fixed angles and distances within the frame of the 3D body scanner.

Before the scanner measurement software can locate body landmarks on a 3D body model, it must find separation points between the arms and the torso, and the legs and the torso. Once the scanner measurement software finds these separation points, it then searches for body landmarks (small of back, back of neck, and many others). Finding these body landmarks, which act as "calibration points" on the 3D body model, determines the location and extraction of various measurements.

If the scanner measurement software fails to produce a 3D body model, an error message will appear on the computer display. This can occur if the individual stands in the scan chamber with legs not separated, or their arms too close to their torso.

Failure to create a 3D body model can also occur if the individual within the scan chamber is in motion during the scan acquisition.

However, there are instances where the scanning software can be "fooled" into producing an unusable $3 \mathrm{D}$ body model. These instances are rare, but they do occur and no error messages are produced. 
Any overlapping areas, such as the pannus depicted in Figure 8, will distort the body model and render certain measurements invalid.
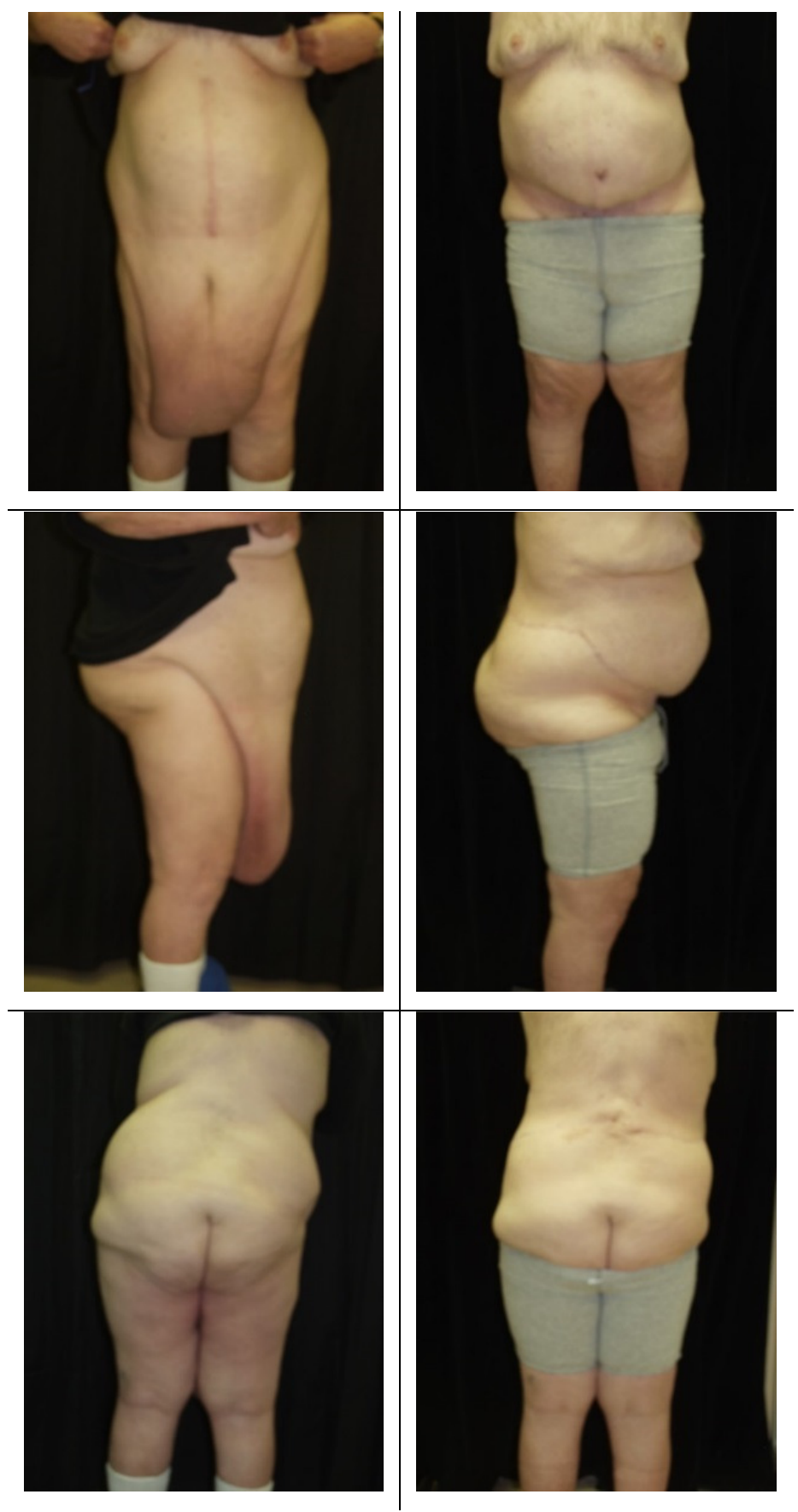

Figure 8. Pre- and Post-Operative Photographs of an Extreme Circumferential Pannulectomy

Figure 9 displays the pre-operative 3D body model, shown in "point-cloud" mode, and the results of applying the lower body measurement template. 


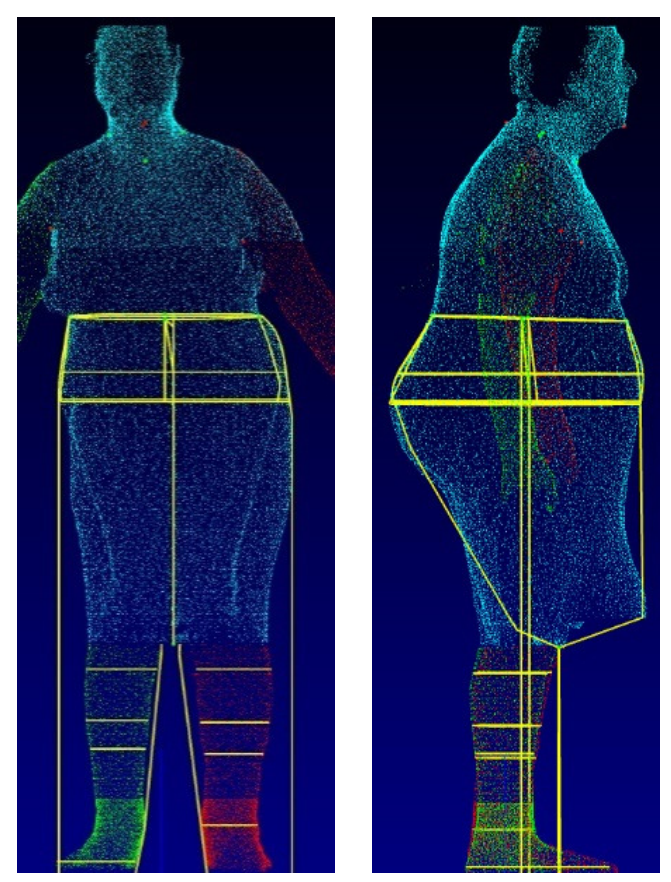

Figure 9. Pre-operative 3D Body Model of Patient with Lower-Body Measurement Template Applied

Note that the measurement software treats the pannus as part of the torso (blue). In this case, the right and left thigh measurements are completely inaccurate as the scanner measurement software searches for the separation between the legs and the torso, then works its way down to the maximum circumference of each thigh. The thigh measurements can be seen here as top lines on the left (represented as green) and right (represented as red) legs.

Figure 10 depicts the 3D body model, with measurement lines, of the post-operative patient.
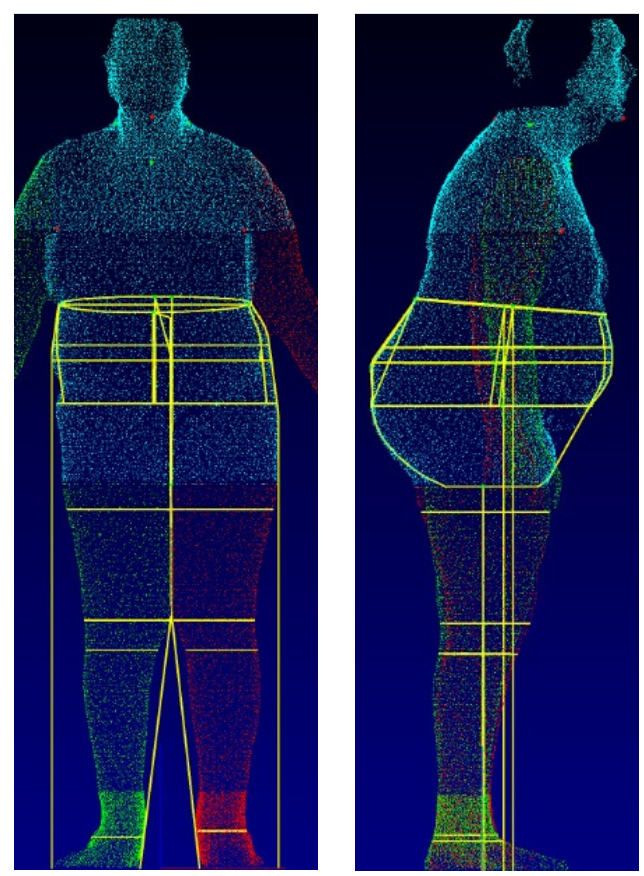

Figure 10. Post-Operative 3D Body Model Images - Correct Location of Thigh and Torso Separation

The removal of the pannus eliminated the overlap between the torso and the legs. The scanner software located the correct point of separation between the legs and the torso, and the thigh measurements extracted by the lower-body measurement template appear accurate. 


\section{Case 2. Gluteal Implants}

Gluteal implants are generally carved blocks of material designed to be implanted within the buttocks, under the gluteal muscles. The result desired is a fuller, rounded posterior. The scanning software contains measurements that are of interest to evaluating the pre-operative buttocks area, and documenting the physical changes to the body post-surgery.

Figure 11 depicts the pre- and 4-month postoperative 3D body models of a patient who underwent a gluteal implant procedure. The measurement lines associated with applying the lower-body measurement template are present. The images are shown in "surface" mode.
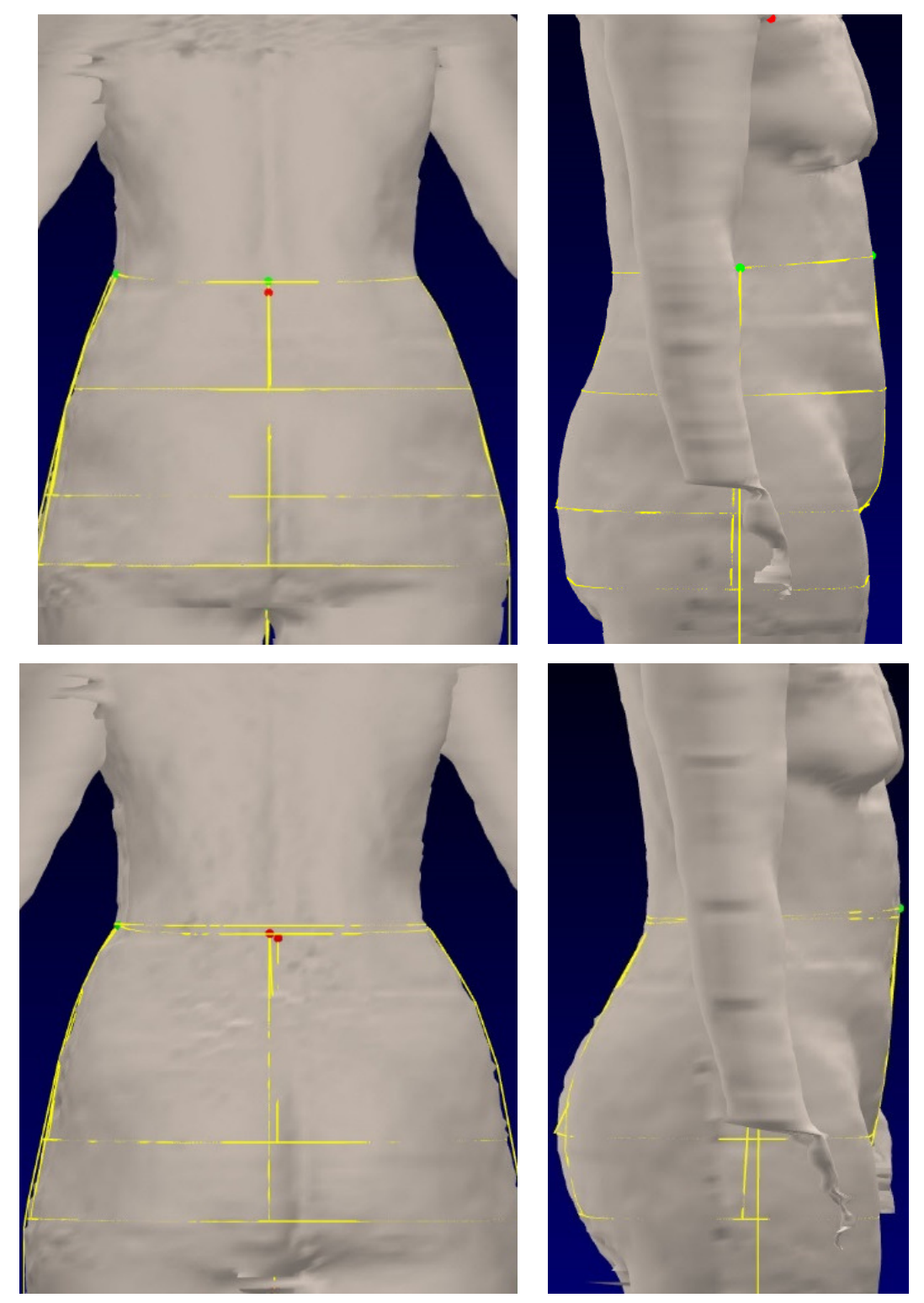

Figure 11. Pre- and Post-operative 3D Body Model Images from a Gluteal Implant Procedure

The post-operative buttocks area has a rounded appearance, and a profile with more pronounced curvature. 
Figure 12 presents the pre- and post-operative measurements extracted from these 3D body models.

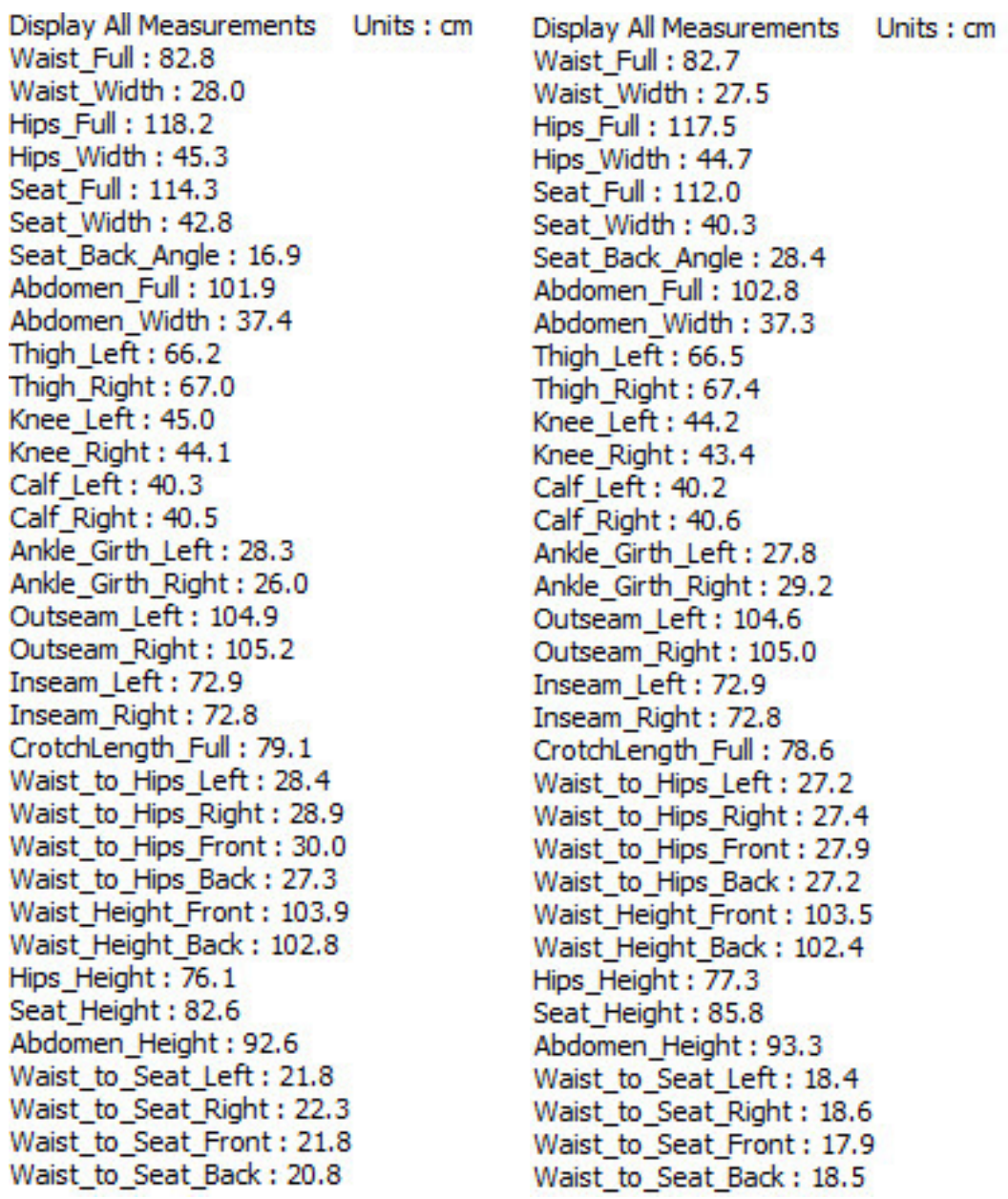

Figure 12. Pre- and Post-Operative Lower Body Measurement Extractions

The result of this "fuller" appearance is captured by the scanner, as it relocated the "Seat_Height" measurement upward. The "Seat_Full" measurement is circumferential. The scanner locates the maximum circumference of the buttocks. The height of this measurement, the "Seat_Height," is given in $\mathrm{cm}$ from the floor.

The post-operative "Seat_Height" is $3.2 \mathrm{~cm}$ above the pre-operative, reflecting the raised curvature that was not present before. The raised height of this point results in a circumferential measurement that does not transverse as much of the hips as before. The "Waist_to_Seat" measurements for Left, Right, Front and Back represent this change of height in terms of linear units.

An interesting measurement to consider is the "Seat_Back_Angle." The "Seat_Back_Angle" is a measurement that extends from the back of the waist point to the point of maximum circumference of the buttocks (Seat_Full). One can observe that the angle has increased post-surgery, reflecting the pronounced curvature of the buttocks from the gluteal implants. 


\section{Case 3. Chest Contouring Procedure}

Chest contouring is a generic term for surgical procedures affecting the shape of male chest. Such procedures include gynecomastia (reduction of excessive breast tissue) and pectoral implants, among others.

Figure 13 depicts an artist's rendering of the steps involved in a gynecomastia procedure.
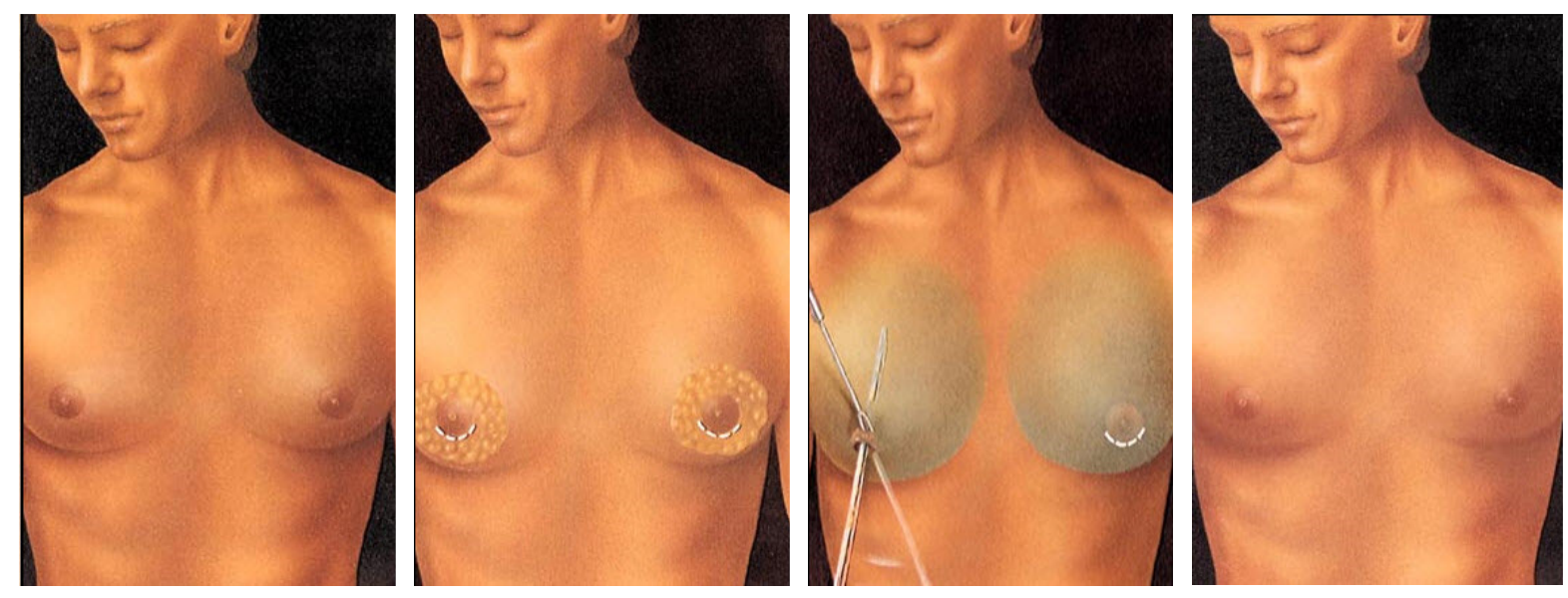

Figure 13. Artist's Rendering of a Gynecomastia Procedure Using Liposuction

In the artist's rendition, the surgeon makes an incision under each lower areola and inserts a cannula for removal by breakage and suction of the excess lipids.

Figure 14 presents an individual who underwent a gynecomastia procedure. In this case, the surgeon elected remove and reconstruct part of the chest area, including the relocation and resizing of the nipple complex.
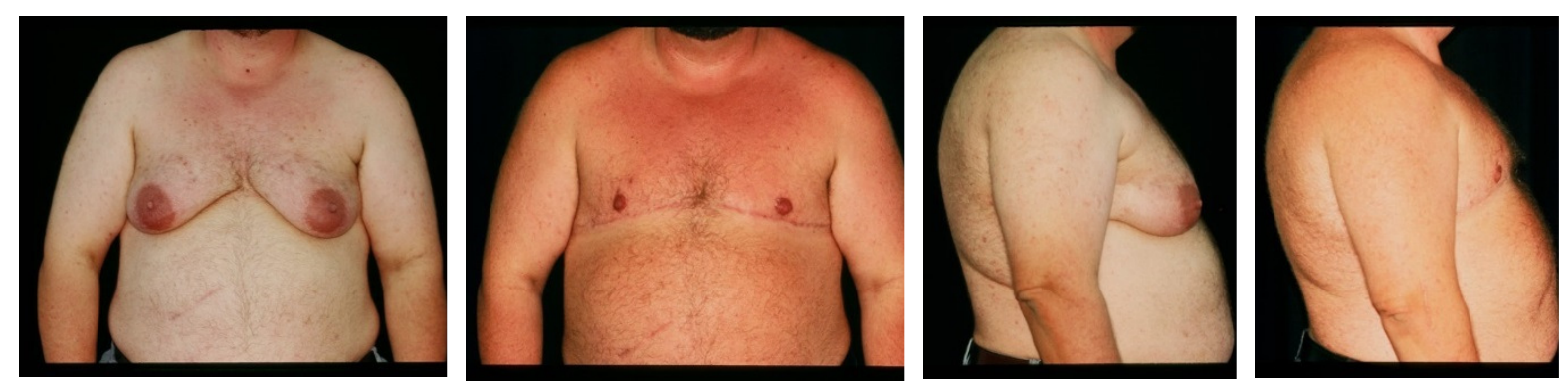

Figure 14. Gynecomastia Procedure with Surgical Tissue Removal and Nipple Complex Relocation

The 3D body scanner offers excellent surface coverage for the front of the torso and can be utilized to measure and evaluate the pre-operative gynecomastia patient. Post-operative scanning can be used to document the results of the procedure.

Figure 15 displays the 3D body model pre-operative front and side views juxtaposed with the postoperative front and side views of a patient who underwent a gynecomastia procedure. The images are shown in "surface view." The yellow lines represent measurements that have been extracted by applying a measurement template programmed to produce measurements of the bust area. This is the same template used to evaluate breast augmentation and breast reduction patients. The reader is encouraged to refer to the reference section for papers on the application of 3D body scanning to breast augmentation and breast reduction procedures. 

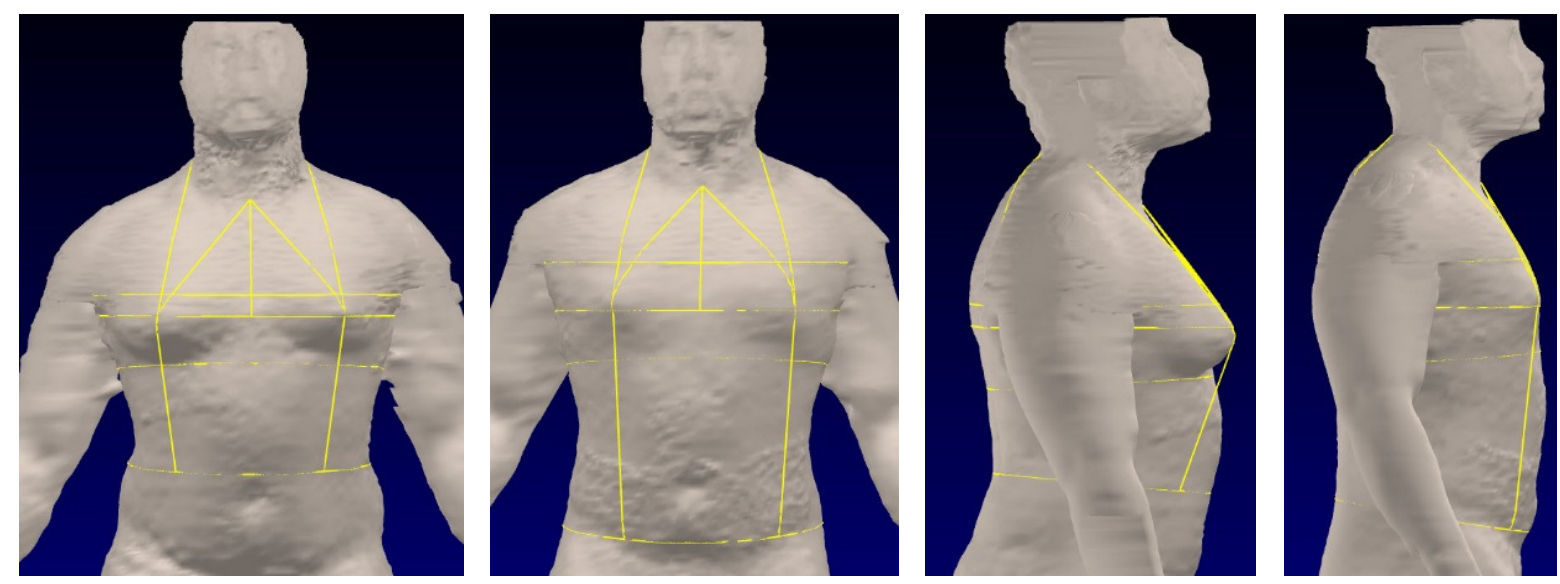

Figure 15. Pre- and Post-Operative 3D Body Model Images of a Gynecomastia Procedure

Figure 16 presents the pre- and post-operative measurements extracted by the measurement profile applied to each 3D body model.

\begin{tabular}{|c|c|}
\hline $\begin{array}{l}\text { Display All Measurements Units : cm } \\
\text { Bust_Full : } 125.8 \\
\text { Bust_Front : } 67.2 \\
\text { Bust_Back: } 58.6 \\
\text { Bust_Width : } 39.9 \\
\text { Bust_Full(Contoured) : } 126.8 \\
\text { Bust_Height : } 128.1 \\
\text { Chest_Full : } 123.8 \\
\text { Chest_Height : } 130.9 \\
\text { Chest_Width : } 40.5 \\
\text { Underbust_Full : } 102.7 \\
\text { Underbust_Front : } 54.6 \\
\text { Underbust_Back : } 48.2 \\
\text { Underbust_Width : } 35.0 \\
\text { Underbust_Height : } 122.1 \\
\text { FrontNeck2Bust_Left : } 22.5 \\
\text { FrontNeck2Bust_Right : } 22.4 \\
\text { SideNeck2Bust_Left : } 27.6 \\
\text { SideNeck2Bust_Right : } 27.6 \\
\text { Neck2BustLine_Front : } 18.8 \\
\text { Neck2BustLine_Back : } 23.0 \\
\text { Waist_Full : } 95.0 \\
\text { BustToWaist_Left : } 21.6 \\
\text { BustToWaist_Right : } 21.8 \\
\text { Bust2Bust_Horizontal : } 24.7 \\
\text { Bust_Prominance_Left : } 34.0 \\
\text { Bust_Prominance_Right : } 34.1\end{array}$ & $\begin{array}{l}\text { Display All Measurements Units : cm } \\
\text { Bust_Full : } 114.3 \\
\text { Bust_Front : } 63.5 \\
\text { Bust_Back : } 50.8 \\
\text { Bust_Width : } 37.6 \\
\text { Bust_Full(Contoured) : } 114.9 \\
\text { Bust_Height : } 126.9 \\
\text { Chest_Full : } 113.3 \\
\text { Chest_Height : } 133.1 \\
\text { Chest_Width : } 39.8 \\
\text { Underbust_Full : } 100.1 \\
\text { Underbust_Front : } 56.3 \\
\text { Underbust_Back : } 43.8 \\
\text { Underbust_Width : } 34.0 \\
\text { Underbust_Height : } 120.9 \\
\text { FrontNeck2Bust_Left : } 22.0 \\
\text { FrontNeck2Bust_Right : } 21.8 \\
\text { SideNeck2Bust_Left : } 26.3 \\
\text { SideNeck2Bust_Right : } 27.0 \\
\text { Neck2BustLine_Front : } 18.2 \\
\text { Neck2BustLine_Back : } 24.9 \\
\text { Waist_Full : } 97.0 \\
\text { BustToWaist_Left : } 29.9 \\
\text { BustToWaist_Right : } 30.1 \\
\text { Bust2Bust_Horizontal : } 24.0 \\
\text { Bust_Prominance_Left : } 31.4 \\
\text { Bust_Prominance_Right : } 32.0\end{array}$ \\
\hline
\end{tabular}

Figure 16. Pre- and Post-Operative Measurement Extractions

One can observe that the bust circumference has been reduced by $11.5 \mathrm{~cm}$. The "bust_contoured" measurement is similar to the bust circumference measurement, except that it follows along the contours of the bust. This has also been reduced proportionately. The difference between the postoperative "Bust_Full(Contoured)" and "Bust_Full" measurements is $0.6 \mathrm{~cm}$.

The pre-operative "Bust_Prominance" measurements indicate that the size of each male breast was similar. The "FrontNeck2Bust" measurements indicate that the nipple location of each male breast were equidistant from the suprasternal notch.

The post-operative measurements reflect the reduction in size of the male breasts, while maintaining symmetrical appearance. 


\section{Case 4. Brachioplasty}

A Brachioplasty is a surgical procedure to reduce/remove pendulous skin from the underarms. Skin laxity occurs with age, from massive weight loss over a short period, or deterioration of muscle tone.

FIGURE 17 outlines the typical markings on the arm that the surgeon might make prior to the brachioplasty procedure. The image on the right is the pre-operative marking of a patient about to undergo the procedure.

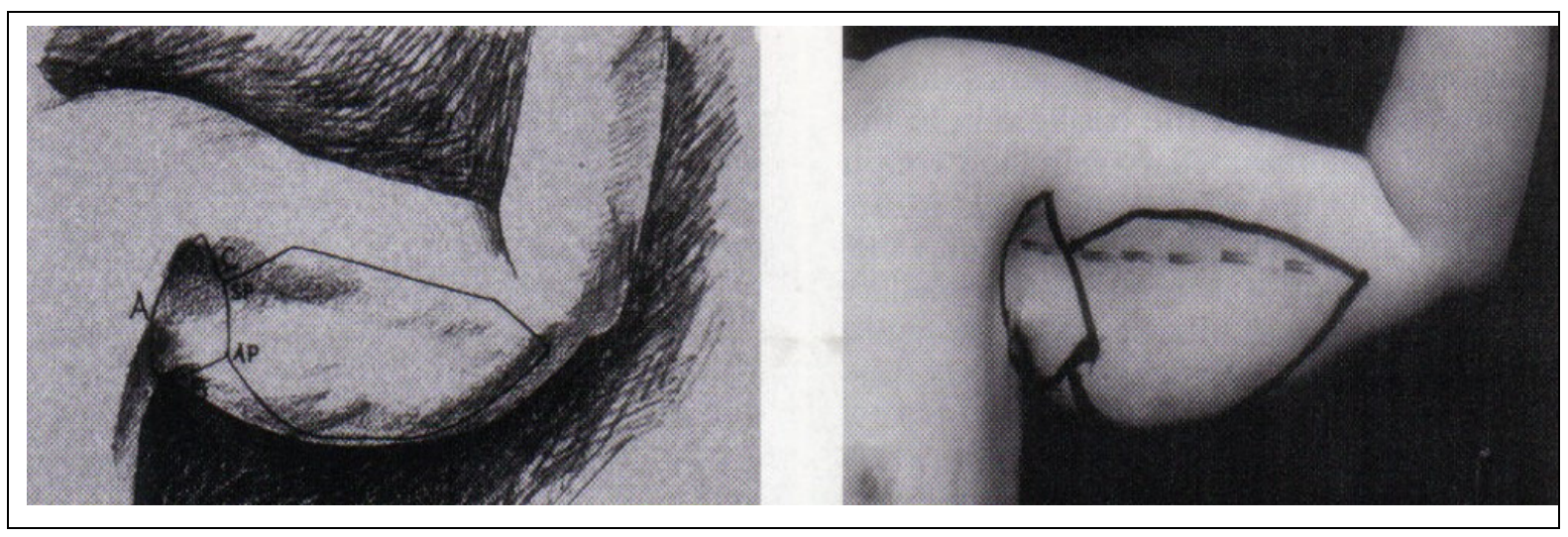

Figure 17. Markings Outlining the Area for a Brachioplasty Procedure

Figure 18 displays pre- and post-operative photographs of a patient that underwent a brachioplasty procedure.

Note that the intent of these "before" and "after" photographs are to highlight the results of the surgery, no measurement information can be gleaned. This is the traditional "2D" method of archiving images of patients. 3D whole-body scanning allows measurements to be taken from the 3D body model, but the 3D body model must be created from a fixed position and posture. It is doubtful whether a 3D body model could be accurately generated from an individual in the scan chamber having a similar pose during scan acquisition as that present in these photographs.
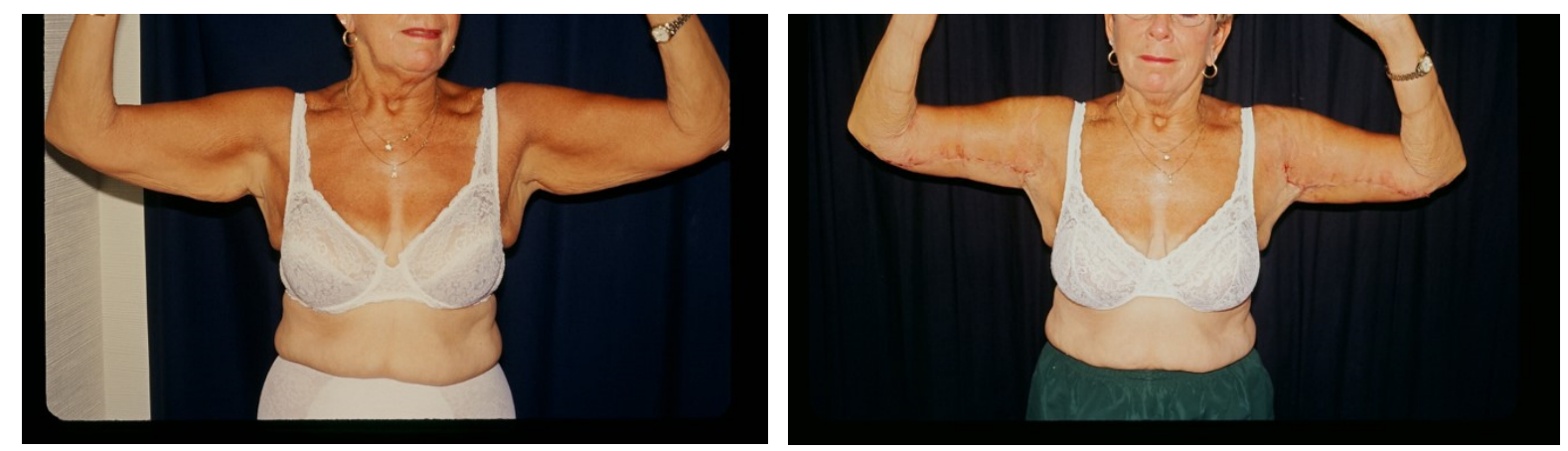

Figure 18. Pre- and Post-Operative Photographs of a Brachioplasty Procedure

Liposuction of this area is not usually a complete solution due to skin laxity. Consequently, the surgical procedure often results in significant scarring, as indicated by the post-operative photograph presented as Figure 19.

A scar on this area, that is, the inner upper arm, will not be represented well on the 3D body model as this area of the individual's body within the scan chamber is not directly exposed to the sensors. 


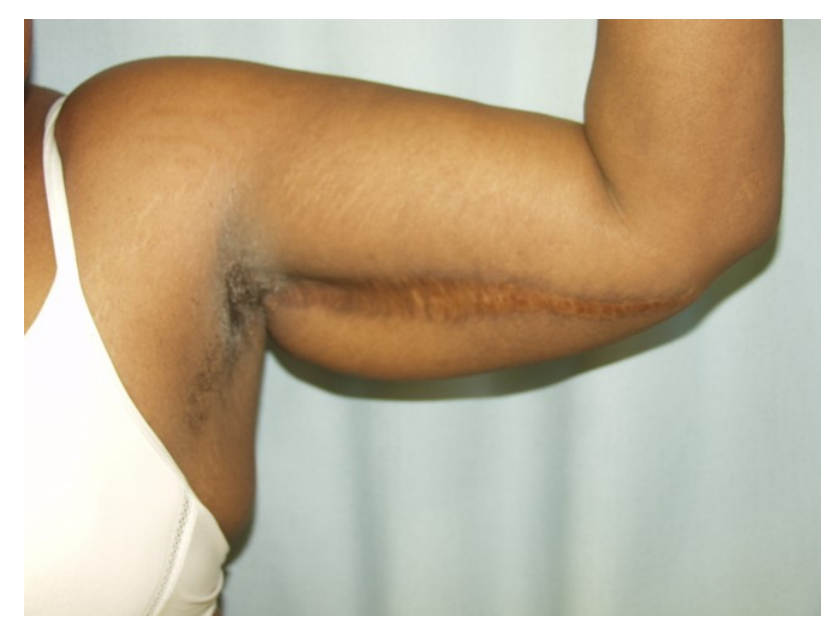

Figure 19. Scar resulting for Brachioplasty Procedure Not Well-Represented on 3D Body Model

This scarring pattern closely follows the dotted marking depicted in Figure 17.

We have seen how the coverage area of the $3 D$ scanner determines its efficacy in creating a measurable 3D body model from the individual standing in the scan chamber. The 3D body scanner purposely distorts the head, hands and feet.

We have also observed that the scanner offers excellent coverage of the torso area, including the buttocks, the mid-section of the body, and the legs. The coverage of the arms, we have found, varies from "usable" to poor. This depends on the position of the arms during scan acquisition, as well as the size of the arms.

The quality of the 3D body image produced by the 3D body scanner depends on three factors. The first factor is the position of the individual within the scan chamber. The individual must stand erect, and have their legs sufficiently separated. Inside the scan chamber are two arm rests. These can be elevated by the individual to a position that attempts to ensure separation between the arms and the torso.

We have remarked that separation of the legs and arms from the torso are critical in the formation of a measurable 3D body model. The separation of the legs is straightforward. The floor of the scanner has two footprints that are spaced apart. The individual stands over these footprints in the appropriate direction. In certain cases of the morbidly obese, care is taken by the scanner operator to ensure that the legs are sufficiently separated, often by extending their feet outside of the footprint guidelines. This exposes the legs, which are generally straight, to the light projected by the sensor heads located in the lower half of the scan chamber. This light is reflected into the cameras, resulting in surface coverage of most, if not all or the inner surface areas of the thighs, knees and calves.

Coverage of the arms is often variable. This is true even if the arms are separated wide from the torso. Because the arm handles are elevated by the individual, there is always the possibility that the arms will be extended into a position where the elbows are angled. This can happen when the individual is of short stature. Subsequent 3D body scans of the same individual can result in 3D body models with the arms in a different position, while the leg separation remains consistent.

The software measurement algorithm attempts to find landmarks on each arm, but can be fooled by the position of the elbows.

This is further exacerbated by individuals that present for a brachioplasty procedure. These individuals must often extend their elbows to ensure that the arms are separated from their torso. If the arms are not separated sufficiently, the scanner software will often combine the deltoid and upper arms with the torso, and arm measurements extracted from such a 3D body model will be grossly inaccurate, if the 3D body model is even produced. 
Figure 20 displays images of pre- and post-operative 3D body models of a patient who underwent a brachioplasty procedure. The images are shown in "point-cloud" mode, and the yellow measurement lines represent the circumferential measurements extracted from the 3D body models by applying a measurement extraction profile.

The point-cloud viewing mode is a representation of the collection of points created by the scanning process that comprise the surface of the 3D body model. This display mode allows one to observe circumferential and width measurements readily, as indicated by the yellow measurement extraction lines.
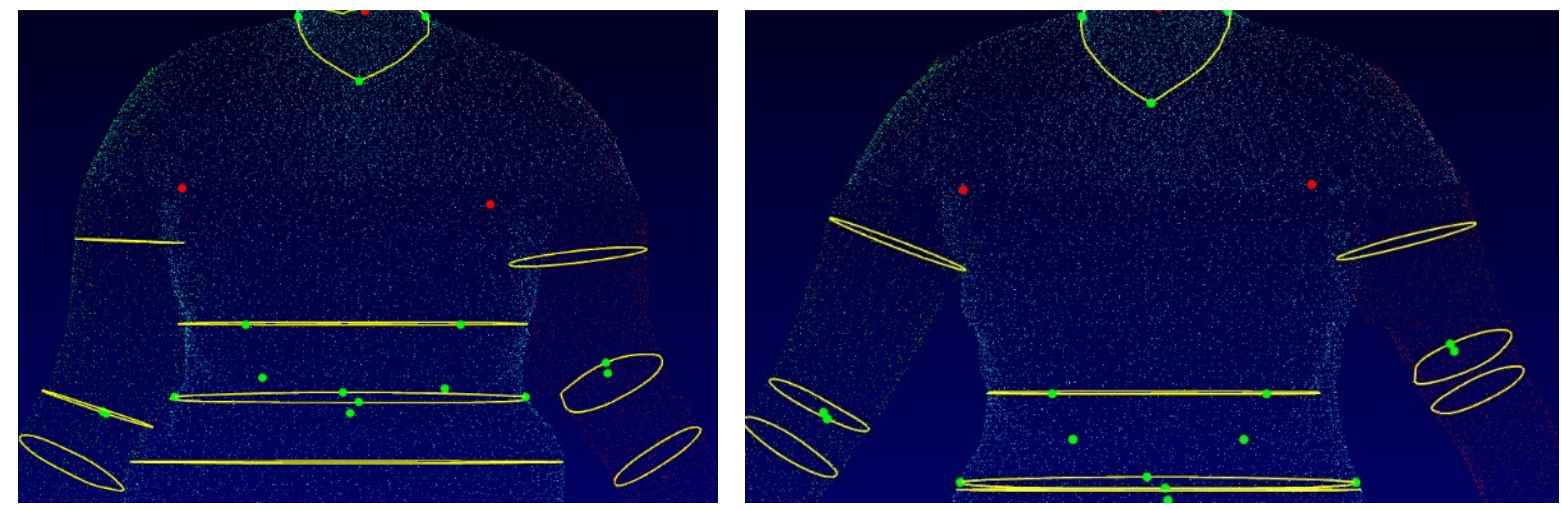

Figure 20. Circumferential Measurements of the Bicep, Elbow and Forearm

Once can observe the circumferential measurements associated with the arms. These are the biceps, elbow and forearm, respectively. The position of the arms is slightly different between each scan.

Figure 21 presents the extracted measurements from each 3D body model.
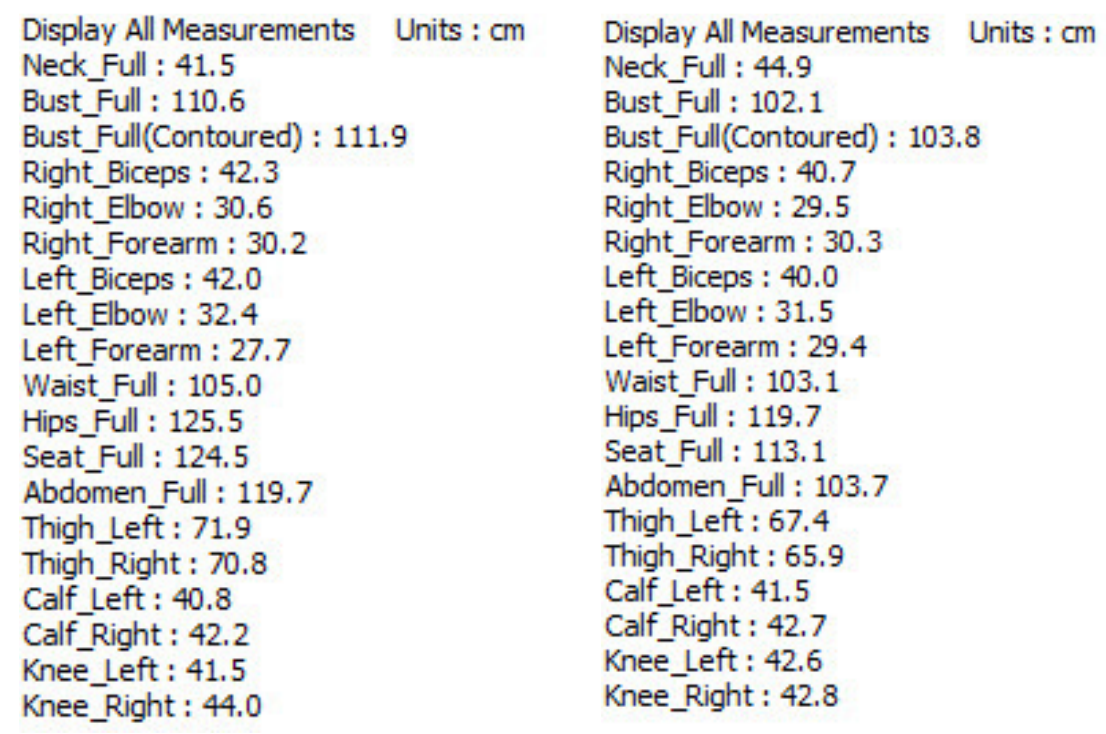

Figure 21. Pre- and Post-Operative Measurement Extraction for Brachioplasty Procedure

The brachioplasty resulted in a reduction in the circumference of the right bicep by $1.6 \mathrm{~cm}$, and the circumference of the left bicep by $2.0 \mathrm{~cm}$.

Due to the different positions of the arms during each scan, the measurements extracted from the 3D body models are better interpreted as relative changes rather than absolute changes between these circumferential measurements. 


\section{A Brief Discussion on 3D Body Scanning and Lipoplasty}

Liposuction procedures must be treated in a different manner. This is because liposuction is often an adjunct procedure, complementing other surgeries such as an abdominoplasty. Liposuction is often used as the sole body contouring procedure. The results of the lipoplasty procedure are not immediate, as edema is usually present. The use of a 3D body scanner and its measurement software can document the pre-operative body, and the "area of interest" with fidelity. A post-operative scan acquired shortly after surgery will have measurements that reflect the extent of the edema, and not the eventual changes to the body contours resulting directly from the lipoplasty procedure. Periodic post-operative scanning can document the reduction in edema. It has been our experience that edema usually abates six months after the lipoplasty procedure. Body scans acquired after this period can therefore be utilized for evaluating post-operative contour changes. However, care must be taken in assessing these measurement changes as the patient may have started an exercise or weight loss regimen in the interim between the first post-operative 3D scan and 3D scans acquired some months after surgery. Circumferential measurements appear to be less useful than linear contour measurements, depending on the area of the body affected by the lipoplasty procedure. Regardless of edema abatement or postoperative fitness or diet changes, post-operative 3D scans accurately reflect the body dimensions at the date and time of acquisition and can be stored in the patient's digital archive.

\section{Summary}

The effectiveness of a 3D body scanner as an aid to measuring body contours for the procedures discussed above comes down to adequate surface coverage of the individual within the scan chamber. The 3D body scanner can't create an accurate image of areas of the body that its sensors are unable to reach.

In certain instances, such as the extreme circumferential pannulectomy where the pannus overlapped the waist and the front thigh area, the scanner software was unable to distinguish the separation between the torso and the legs, resulting in a 3D body model that was in error.

The 3D body scanner utilized was a whole-body, booth type scanner. It displayed adequate to excellent and consistent coverage for the torso, front and back, including the buttocks, and the legs. Provided that the subject wasn't moving during the scan acquisition process, desired measurements can be extracted that appear faithful and accurate for these areas of the body.

The 3D body scanner has proved to be useful for documenting pronounced changes to the body resulting from a surgical procedure. Breast augmentation, breast reduction, abdominoplasty, thigh lift, gluteal and calf implants, gynecomastia and circumferential pannulectomy procedures can benefit from the 3D scanner and the appropriate measurements that the scanner software can extract. This is helpful for pre-operative evaluation, and post-operative validation.

The 3D body scanner's coverage of the arm area appears to be inconsistent. This is partly due to the position of the arms during scan acquisition, which can vary from scan to scan, and partly due to lack of sensor coverage for the portion of the arms near the torso. Separation of the arms by the patient is sometimes difficult depending on their size. The arms must be kept motionless during the scan acquisition. Though this process only takes several seconds, the pendulous skin overhang on some of the patients' arms are often in motion during this process.

In such situations, the circumferential measurements of the arms extracted by the scanner's measurement software can be inaccurate, making the 3D body scanner less useful for evaluating or validating a surgery such as brachioplasty.

Figure 22 presents a table enumerating the surgeries conducted that have utilized the 3D whole-body scanner to acquire and measure pre-operative patients. The classification of effectiveness is the surgeon's own opinion. The "Head, Hand, Feet" entry is simply a reminder that the scanner coverage is not focused on these areas and as a result, 3D body scans were not acquired for patients that underwent any cosmetic surgery procedures to these regions. 


\begin{tabular}{|l|c|c|c|}
\hline \multicolumn{1}{|c|}{ Surgical Procedure } & Effective & Marginal & Not Useful \\
\hline Head, Hand, Foot & & & X \\
\hline Arms & & $X$ & X \\
\hline Neck & & & \\
\hline Scar Revision & & & \\
\hline Hidden Genitals & $\mathrm{X}$ & & \\
\hline Breast Augment & $\mathrm{X}$ & & \\
\hline Breast Reduction & $\mathrm{X}$ & & \\
\hline Abdominoplasty & $\mathrm{X}$ & & \\
\hline Lower-Body Lift & $\mathrm{X}$ & & \\
\hline Gluteal Implant & $\mathrm{X}$ & & \\
\hline Chest Contouring & $\mathrm{X}$ & & \\
\hline Pannulectomy & $\mathrm{X}$ & & \\
\hline Mastopexy & $\mathrm{X}$ & & \\
\hline Breast Reconstruct & $\mathrm{X}$ & & \\
\hline Pectoral Implant & & & \\
\hline Thigh Lift & & & \\
\hline Lipoplasty & & & \\
\hline
\end{tabular}

Figure 22. One Surgeon's Opinion as to the Applicability of the $3 D$ Body Scanner to Various Cosmetic Surgery Procedures.

\section{References}

Integrating a 3D Body Scanner into an Active Bariatric Surgery Clinic: Practical Experiences, History, Tips and Pitfalls. David Stefan, M.S.E.E., David A. Gilbert, M.D., Stephen D. Wohlgemuth, M.D. Paper ID 562014 3D Body Scanning Conference. Lugano, CH. October 2014.

This paper details the history of the $3 D$ whole-body scanners employed by The Hague Center for Cosmetic Surgery. It includes a discussion of the many issues that needed to be resolved before $3 D$ scanning of patients became integrated into the workflow of the clinic's personnel. There is a discussion of best practice protocols that are in use for the 3D scanner in a clinical setting.

Breast Augmentation Virtual Surgery Using 3D Body Scanning: Bridging the Gap between Patient Expectations and Surgical Practicalities. David A. Gilbert, M.D. Paper ID 552013 3D Body Scanning Technologies, Long Beach, CA, USA. November 2013.

This paper details the early efforts to develop an effective measurement template for use on breast augmentation and breast reduction patients. It also gives insight into the ability of the $3 D$ scanner to export 3D body models to CAD programs, and how it is possible to perform "virtual surgery" to accurately predict the outcome of breast augmentation and breast reduction procedures prior to conducting the surgery. 